\title{
Chromosomal organization and evolutionary history of Mariner transposable elements in Scarabaeinae coleopterans
}

\author{
Sarah G Oliveira', Diogo C Cabral-de-Mello ${ }^{2}$, Rita C Moura ${ }^{3}$ and Cesar Martins ${ }^{1 *}$
}

\begin{abstract}
Background: With the aim to increase the knowledge on the evolution of coleopteran genomes, we investigated through cytogenetics and nucleotide sequence analysis Mariner transposons in three Scarabaeinae species (Coprophanaeus cyanescens, C. ensifer and Diabroctis mimas).

Results: The cytogenetic mapping revealed an accumulation of Mariner transposon in the pericentromeric repetitive regions characterized as rich in heterochromatin and $C_{0} t-1$ DNA fraction (DNA enriched with high and moderately repeated sequences). Nucleotide sequence analysis of Mariner revealed the presence of two major groups of Mariner copies in the three investigated coleoptera species.

Conclusions: The Mariner is accumulated in the centromeric area of the coleopteran chromosomes probably as a consequence of the absence of recombination in the heterochromatic regions. Our analysis detected high diversification of Mariner sequences during the evolutionary history of the group. Furthermore, comparisons between the coleopterans sequences with other insects and mammals, suggest that the horizontal transfer (HT) could have acted in the spreading of the Mariner in diverse non-related animal groups.
\end{abstract}

Keywords: Chromosomal rearrangements, Evolution, Heterochromatin, Horizontal transfer, Repetitive DNA, Transposition

\section{Background}

The repetitive DNAs represent a significant fraction of eukaryotic genomes and are primarily enriched in the heterochromatic regions, although some of them were observed in euchromatic regions [1-4]. Among the repeated DNAs, the transposable elements (TEs) are DNA sequences capable of changing their location in the genome, moving from one site to another, which seems to benefit only the elements and, for a long time, they have been considered as a "parasitic" and/or "selfish" elements. However, TEs represent an evolutionary force that provides the potential conditions for the emergence of new genes, modify gene expression, and adaptation to new environmental challenges [5-7]. In this way, TEs have a major role shaping and influencing the structure and function of the genomes [8].

\footnotetext{
*Correspondence: cmartins@ibb.unesp.br

'Morphology Department, Biosciences Institute, UNESP - São Paulo State University, Botucatu, SP 18618-970, Brazil

Full list of author information is available at the end of the article
}

Among several groups of TEs, Mariner-like elements (MLEs) are a superfamily of DNA transposons that consists in a single gene without introns flanked by two terminal inverted repeat (TIR) of about $30 \mathrm{bp}$, performing a total length of approximately $1.300 \mathrm{bp}$. Each terminal repeat is flanked by a TA dinucleotide, resulted from duplication of the target site duplication (TSD) $[9,10]$. The Mariner transposase gene encodes a protein of 330-360 amino acids, which recognizes the TIRs and cuts both strands at each end, being responsible for transposition by excising, exchanging, and fusing DNAs in a coordinated manner [11,12].

The Mariner superfamily is probably the most widespread and diverse group of TEs found in animals, persisting in the genomes through evolutionary time [13]. The MLE history started with their discovery in insects (being observed in several orders), and now their distribution has been reported in multiple invertebrate and vertebrate genomes $[9,14-16]$. The high similarity between sequences from distantly related organisms, the 
incongruence between TE and phylogeny, and the unequal distribution of some Mariner subfamilies among closely related taxa indicate that the horizontal transfer (HT) contributed to this widespread distribution [17-20].

The combination of molecular and cytogenetic analyses has established that several transposable elements are associated with chromosomal rearrangements such as deletions, duplications, inversions, the formation of acentric fragments and dicentric chromosomes, recombination and translocations of host genomes. In Drosophila, species in which there are a variety of studies, two kinds of elements, $P$ and hobo, are especially prone to induce chromosome rearrangements. However, other transposons also appear to mediate chromosome rearrangements; these include the elements BEL, $H e T-A$, Mariner, roo, Tango and TART [21-23].

The cytogenetic mapping of repetitive DNAs has improved the knowledge of genome organization and chromosomal differentiation during the evolutionary history of the species. On the other hand, the genome organization of repetitive DNAs has been poorly investigated in Coleoptera, with only one study involving the cytogenetic mapping of TEs [24]. With the aim to contribute to the knowledge of coleopteran genomes evolution at molecular and chromosomal level, we investigated the repetitive DNA fraction of three Scarabaeinae species
(Coprophanaeus cyanescens, C. ensifer and Diabroctis mimas), cytogenetically characterized by the presence of large blocks of heterochromatin [19,25]. The subfamily Scarabaeinae comprises a diverse and cosmopolitan group of Coleoptera that play an important role in the conservation of ecosystems as seed dispersers, pollinators, and recyclers of organic matter [26]. The overall chromosomal distribution of repetitive DNAs was investigated through the chromosomal hybridization of $C_{0} t-1$ DNA fraction (DNA enriched with high and moderately repeated sequences), and the genomic features of Mariner TEs were addressed through nucleotide sequencing and chromosomal mapping. The knowledge of the repetitive portion of Scarabainae genomes brings the opportunity to advance in studies of genome organization, species evolution, and chromosome evolution in coleopterans.

\section{Results}

\section{Cytogenetic mapping of $C_{o} t-1$ DNA and Mariner}

The $C_{0} t-1$ DNA fraction was isolated from each genome of C. cyanescens, C. ensifer and D. mimas, and hybridized to their own chromosomes (Figure $1 \mathrm{~A}-\mathrm{C}$ ). This $C_{0} t-1$ DNA revealed positive hybridization in the long arms of all autosomes and $\mathrm{X}$ chromosome of the two Coprophanaeus species, and in the long arm of the Y chromosome of $C$. ensifer (Figure 1A, B). The $C_{0} t-1$ DNA fraction
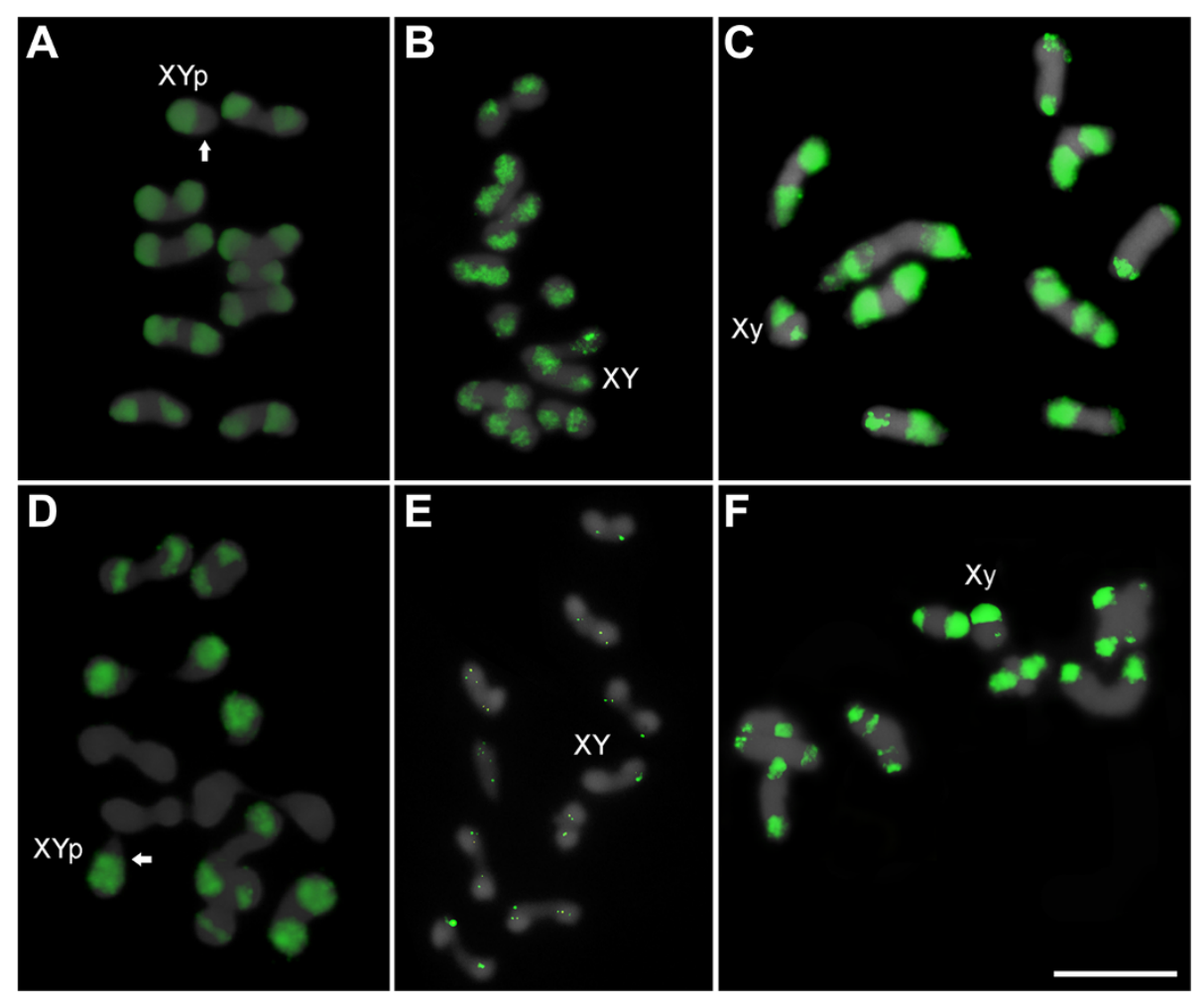

Figure 1 Metaphases I of Coprophanaeus species submitted to FISH. The metaphases of $C$. cyanescens $(\mathbf{A}, \mathbf{D})$, C. ensifer (B,E) and Diabroctis mimas $(\mathbf{C}, \mathbf{F})$ were probed with $C_{0} t-1$ DNA $(\mathbf{A}-\mathbf{C})$ and Mariner transposable element (D-F). The arrows indicate the chromosome $\mathbf{Y}(\mathbf{A}, \mathbf{D})$. Bar $=5 \mu \mathrm{m}$. 
hybridization in D. mimas evidenced large pericentromeric blocks in all autosomal pairs and in the sex chromosomes, extending to the short arms of some autosomal chromosomes (never to the long arm), including the terminal region, and in the terminal region of one autosomal pair (Figure 1C). Cross-species hybridization of $C_{0} t-1$ DNA fraction showed positive hybridization only among species of the same genus, showing the same pattern observed for hybridization of probe originated from the same genome (as shown in Additional file 1: Figure S1). These patterns of $C_{0} t-1$ DNA hybridization were similar to the data previously generated by $\mathrm{C}$-banding on the three species [25,27]. However, it was not observed $C_{0} t-1$ DNA hybridization in the $\mathrm{C}$-positive banded centromeric region of the $\mathrm{Y}$ chromosome of $C$. cyanescens, as well as in the interstitial blocks of the short arms of three autosomal pairs and in the telomeric block in a small autosomal pair observed in C. ensifer [27]. Otherwise, $C_{0} t-1$
DNA blocks observed in the terminal region of an autosomal pair and in the centromeric region of the $\mathrm{Y}$ chromosome of $D$. mimas were not observed by C-banding (Figure 2, [25]).

The FISH using probes of Mariner sequences in $C$. cyanescens labeled the $\mathrm{X}$ and $\mathrm{Y}$ sex chromosomes and four autosomal pairs with large pericentromeric blocks and three large autosomal pairs with pericentromeric labeling that also cover the long arm (Figure 1D). In $C$. ensifer the mapping of Mariner revealed small pericentromeric blocks in the $\mathrm{X}$ and in all autosomal chromosomes (Figure 1E). In D. mimas the pattern was similar to the obtained by $C_{0} t-1$ DNA hybridization, however, the blocks observed were smaller (Figure 1F). A schematic ideogram showing the distribution of heterochromatic regions revealed by $\mathrm{C}$-banding, and fluorescent in situ hybridization with $C_{0} t-1$ DNA and Mariner sequences probes is presented in Figure 2.

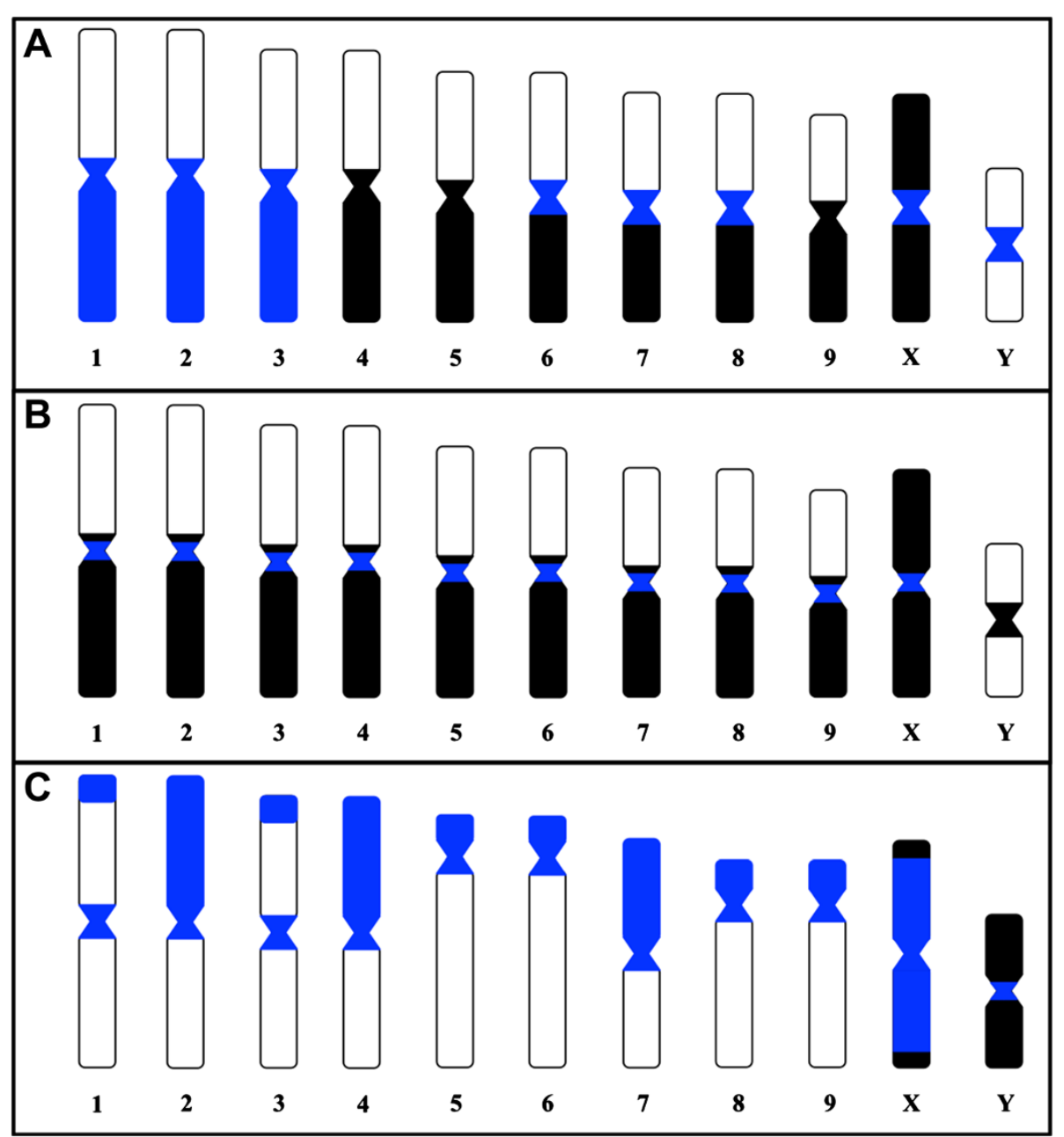

Figure 2 Schematic ideogram showing the hybridization patterns described for Coprophanaeus cyanescens (A), C. ensifer (B) and D. mimas (C). Black represents the distribution of heterochromatin revealed by C-banding according to previous works $[25,27]$ and $C_{0} t-1$ DNA. Blue represents the distribution of Mariner elements. 


\section{Analysis of Mariner sequences}

Nucleotide transposase (partial DDE domain - region with three conserved aminoacid: Asparagine-AsparagineGlutamine) sequences of approximately 230 bp were obtained for C. cyanescens (eight sequences), C. ensifer (six sequences) and D. mimas (nine sequences) (Additional file 2: Dataset S1). The comparative analysis of Mariner to several vertebrates and invertebrates showed that the Mariner sequences are organized into two major groups (I and II), and the group I is subdivided into 3 branches

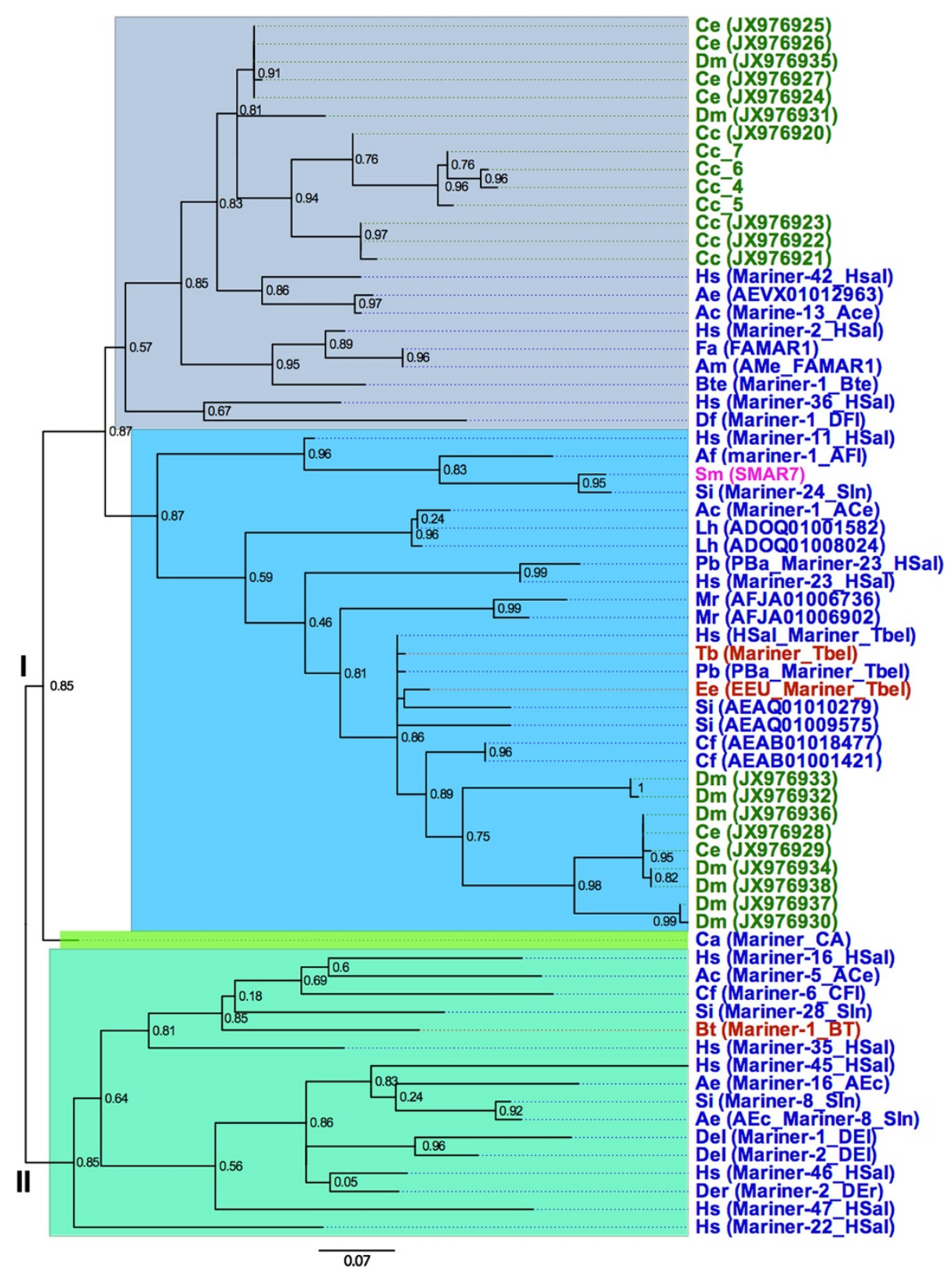

Figure 3 Alignment guide tree of Mariner families. The sequences obtained for beetles in this work are indicated in green. Except individual sequences with accession numbers provided, all other sequences are represented by consensus sequences deposited in Repbase, with their Repbase ID. The sequences are indicated in different colors considering if they are derived from mammals (red), planaria (pink), beetles (green) and other insects (blue). Species are Cc (Coprophanaeus cyanesces), Ce (C. ensifer), Dm (Diabroctis mimas), Ac (Atta cephalotes), Ae (Acromyrmex echinatior), Af (Apis florea), Am (Apis mellifera), Bte (Bombus terrestris), Bt (Bos taurus), Ca (Chymomyza amoena), Cf (Camponotus floridanus), Del (Drosophila elegans), Der (Drosophila erecta), Df (Drosophila ficusphila), Ee (Erinaceus europeus), Fa (Forficula auricularia), Hs (Harpegnathos saltator), Lh (Linepithema humile), Mr (Megachile rotundata), Pb (Pogonomyrmex barbatus), Si (Solenopsis invicta), Sm (Schmidtea mediterranea) and Tb (Tupaia belangeri). The sequences Cc_4 to Cc_7 are smaller than 200 bp and do not have GenBank accession numbers. The Mariner sequences are organized into two major groups (I and II, as indicated), and the groups are subdivided into branches (colored blocks). The branch support values are indicated on the nodes. The scale bar indicates the genetic distance. 
(Figure 3, Additional file 3: Figure S2). In the first branch, there is only the distribution of insect sequences, represented by flies (Drosophila ficusphila), ants (Harpegnathos saltator), bees (Apis florea, Apis mellifera), earwig (Forficula auricularia) and beetles (C. cyanescens, C. ensifer, D. mimas). In the second branch, it is observed the distribution of mammal sequences (Erinaceus europeus, Tupaia belangeri), planaria (Schmidtea mediterranea), and insects, represented by ants (Atta cephalotes, Harpegnathos saltator, Linepithema humile, Solenopsis invicta), bees (Megachile rotundata) and beetles (C. ensifer, D. mimas). In the third branch, it is observed only one sequence from the fly (Chymomyza amoena). The sequences of the group II are organized in one major branch and contain data of mammal (Bos taurus), and insects represented by flies (Drosophila elegans, Drosophila erecta) and ants (Acromyrmex echinatior, Atta cephalotes, Camponotus floridanus, Harpegnathos saltator, Solenopsis invicta).

In the first branch (group I sequences), the sequences of beetles showed similarity with other insect sequences, although they form a separated branch with high branch support value (0.87). In the second branch, the insect sequences are related to Mariner1_Tbel family from mammals. Even including the beetle sequences, it is clear the high similarity of sequences of the mammalians Erinaceus europeus and Tupaia belangeri with the genome of ants Pogonomyrmex barbatus and Harpegnathos saltator (higher than 92\% compared with Erinaceus europeus, and higher than 98\% compared with Tupaia belangeri) (Additional file 4: Dataset S2). The genetic distance within Mariner1_Tbel sequences between mammals and insects (including beetles) species were relatively low (0.017-0.359\%) (Additional file 4: Dataset S2). In turn, the genetic distances observed within Mariner-1_BT sequences among insect species were 0.258-0.525\% (Additional file 4: Dataset S2).

The Mariner sequences of beetles branched out in two into two groups (seen in the first and second branches of group I, Figure 3). Within the first branch, were obtained sequences from $C$. cyanescens, $C$. ensifer and $D$. mimas. However, within the second branch there were only obtained sequences from species $C$. ensifer and $D$. mimas. The genetic distance between this two beetle groups was relatively high (0.338-0.518).

\section{Discussion}

\section{General aspects of heterochromatin and repeated DNAs organization}

The presence of large blocks of heterochromatin and $C_{0} t-1$ DNA fraction in the studied species suggests the occurrence of amplification of repetitive DNAs and/or heterochromatin transfer between the chromosomes during the karyotype differentiation of species as previously observed in other animals [28-31]. This statement is supported by the common pattern of heterochromatic blocks mainly located in pericentromeric areas in relates species, including coleopterans [32-34]. Most information concerning heterochromatin in coleopterans is focused on the description of chromosomal distribution with few data regarding its molecular content. The $C_{0} t-1$ DNA fraction hybridization showed a general pattern coinciding with the data generated by C-banding [25,27], indicating that the heterochromatin is enriched in highly repetitive DNA. The presence of large blocks of $C_{0} t-1$ suggests an abundance of repetitive sequences, and cross-species hybridization analysis among Phanaeini species evidences high conservation between the fractions of repetitive DNA within genera and divergence between the two different studied genera. However, the use of $C_{0} t-1$ DNA fractions as probes in Dichotomius species (Coleoptera, Scarabaeidae) allowed the observation of heterochromatin distribution patterns highly conserved in the terminal/ sub-terminal region and an extensive variation in relation to the pericentromeric heterochromatin [35]; which contrasts with the Phanaeini species studied. These data reinforce the intense evolutionary dynamics of the repeated DNA fraction by mutation, gene conversion, unequal crossing-over, circular replication and slippage replication [36-38] generating high divergence among taxa above the genus level.

\section{Chromosomal organization of Mariner transposable elements}

It is a common observation that some transposable elements may be overabundant in specific regions of chromosomes, and the results obtained with the mapping of Mariner shows that these sequences are not randomly distributed and have accumulated in the heterochromatic areas. However, the accumulation of this element in euchromatic areas was recently reported in Eyprepocnemis plorans [4]. The accumulation of a large amount of copies in the heterochromatic regions can indicates a selection against insertions of TEs in euchromatin based on ectopic exchanges. Different major forces can affect TEs in heterochromatin and euchromatin regions of the genome, being that accumulation in heterochromatin regions explained by the absence of selection against insertional mutations in genetically inert regions, and stochastic accumulation of deleterious elements in regions with no recombination $[1,2,39]$.

Possibly the absence of labeling in three autosomal pairs of $C$. cyanescens indicates that the evolutionary history of these sequences within the genome of the species follows a distinct pattern; possibly including suppression of recombination between these chromosomes with the other autosomes.

The accumulation of Mariner sequences in the pericentromeric regions is possibly due to the low rate of 
recombination characteristic of these regions, and could indicates that this element is enriched in regions where the damage of its insertion is reduced [22,40]. Although it is not possible to predict the possible role of these elements in Coleoptera they may be involved with the chromosomal rearrangements, as the occurrence of pericentromeric inversion observed in $D$. mimas. This species presents metasubmetacentric (pairs 1, 2, 3 and 7) and acrocentric (pairs 5, 6, 8 and 9) autosomal chromosomes [25], while C. cyanescens and $C$. ensifer have meta-submetacentric morphology for all autosomal chromosomes [27]. In D. mimas the presence of four acrocentric autosomal pairs indicates the occurrence of pericentromeric inversions unlike the standard meta-submetacentric karyotype described for the family Scarabaeidae [41]. Chromosomal rearrangements, as that observed in D. mimas, are possible a consequence of transposable elements, that were reported to be involved with various types of rearrangements by transposition and recombination [42-44].

Another approach to the accumulation of transposable elements is that the Mariner transposon could have been maintained in the pericentromeric region by presenting any functional role in the maintenance of this region [45]. For example, during the evolution of the genome, heterochromatic transposable elements may lose the ability to transpose and accumulate mutations and structural rearrangements, acquiring new functions [46,47]. Feschotte [6] proposed that the movement and accumulation of TEs, as well their derived proteins, have played an important role in the evolution of the genome. The association of TEs and the structure and/or function of centromeres seems to be an usual occurrence, and have been observed in diverse species $[47,48]$.

The mapping of Mariner in the sex chromosomes of the three species could be related to the common spreading of the TEs in most heterochromatic areas of the genome, or the sex chromosomes can act as a refuge for transposable elements as previously reported [49-51]. Several genetic processes can cause an accumulation of TEs in genomic regions where crossing over is reduced or absent [36]. In some cases, for example, the sex chromosomes show the tendency of non-recombining in the genomic regions to accumulate transposable elements [52,53]. Another possibility is that, the recombination suppression itself could inhibit recombination in nearby regions of the sex chromosomes [53].

The transposition/selection model establishes that the distribution and abundance of TEs are indicative of their evolutionary history $[36,54]$. This process involves three stages: (i) invasion of the host genome, (ii) rapid spread by replicative transposition, and (iii) vertical inactivation and accumulation in the heterochromatin. Considering that hypothesis, the Mariner present in C. cyanescens, $C$. ensifer and $D$. mimas could be considered ancient because active and recently acquired elements are expected to be preferentially located in euchromatin. TEs are expected to be overabundant in the heterochromatin where recombination is strongly reduced, and because the TEs cannot be easily removed from heterochromatin once they have been inserted $[55,56]$.

Besides the accumulation in the heterochromatin, the Mariner sequences hybridization patterns are quite different between the three species. This suggests that the chromosomes do not share a general pool of Mariner sequences, and could indicate a different evolutionary path after the emergence within each species.

\section{Mariner transposable elements in Scarabaeinae coleopterans}

The Mariner sequences of Scarabaeinae coleopterans branched out into two groups, showing a relatively high genetic distance between them, indicating an early divergence from an ancestral element. This is consistent with previous studies, proposing that members of the Tc1/ Mariner are probably monophyletic in origin, and diversified in various groups by accumulation of modifications and/or horizontal transfer mechanisms [9,57,58].

Probably, each TE copy of beetles has evolved independently of each other, according to the pattern of molecular evolution related for Mariner transposon. When divergent elements do exist, they display, as observed, a low percentage of similarity to the full-length sequences. This suggests that TEs are highly active within the genome, and that the highly divergent copies reflect relics of ancient mobilizations, as described to Drosophila melanogaster [53].

\section{Mariner horizontal transfer}

Mariner transposable elements have been described in many arthropods, possibly spread by HT [17,20,59]. In general, the phylogenies based on Mariner sequences are not always congruent with the phylogenies of the taxa, suggesting the occurrence of HT [14,60].

The high sequence similarity between sequences from distantly related organisms, the incongruence between TE distribution and phylogeny, and the unequal distribution of some Mariner subfamilies among closely related taxa indicate that the HT contributed to this widespread distribution [18,20,61]. Several TEs have been introduced into mammal lineages through HT [62-65], including Mariner [19,66,67]). Comparative analyses of mammalian genomes show the presence of high amount of TEs, but their content could vary among the different lineages $[18,68]$. The genetic distance within Mariner1_Tbel sequences between mammals and insects species were relatively low, consistent with the phylogenetic distances between them and reinforces the occurrence of HT in the spread of these elements to different taxa [19]. 
Considering the Mariner tree topology clearly indicates the involvement of HT during the evolutionary history of insects and mammals, although it is not possible to show in which evolutionary moment this transfer occurred. Multiple mechanisms may be related to the spread of TE by horizontal transfer, using different types of vectors (external parasites, infectious agents, intracellular parasites and symbionts, DNA viruses, RNA viruses, retroviruses) $[13,69,70]$. Thus, for each described case of a proposed HT, could be implemented a model of transfer. Our results are consistent with the criteria of HT, and reveal interesting patterns of patchy distribution among animals, suggesting a repeated invasion of Mariner from insects to mammals.

\section{Conclusions}

The relatively high genetic distances observed between the two classes of Mariner sequences of beetles, and their distribution in other animals indicate that these two classes had a early origin in the base of insect diversification, or considering the highest similarity for each group with other insect sequences (particularly ants), and also similarity to mammals in one of the groups, there is an evidence that the sequences may have originated by horizontal transfer.

\section{Methods}

\section{Animals and DNA samples}

Adult male samples of Coprophanaeus cyanescens, $C$. ensifer and Diabroctis mimas were collected in Caruaru, Igarassu, Paudalho and Saloá, Pernambuco State, Brazil. The animals were collected in the wild according to Brazilian laws for environmental protection (wild collection permit, MMA/IBAMA/SISBIO n. 2376-1). The experimental research on animals was approved by the ethics committee of Sao Paulo State University (Protocol no. 35/ 08 - CEEA/IBB/UNESP). The testes were fixed in Carnoy solution (3:1 ethanol: acetic acid) and then stored in freezer at $-20^{\circ} \mathrm{C}$. The DNA samples were obtained from living specimens immediately frozen in the freezer at $-20^{\circ} \mathrm{C}$. The procedure for extraction of genomic DNA followed, with minor modifications, the protocol described by Sambrook and Russel [71]. The quality and quantity of purified DNA were analyzed under electrophoresis and spectrophotometry.

\section{$C_{0}$ t-1 DNA preparation}

$C_{0} t-1$ DNA fractions were obtained from C. cyanescens, $C$. ensifer and D. mimas based on reassociation kinetics proposed by Zwick et al. [72] and the modifications described by Ferreira and Martins [73]. The $C_{0} t-1$ DNA fractions obtained were labeled and used directly as probes for chromosome hybridization, being performed hybridizations within the same species and between different species.

\section{Isolation and characterization of Mariner TEs}

The Mariner transposable elements were isolated thought polymerase chain reaction (PCR) with the set of primers MAR-188 F (5' ATC TGR AGC TAT AAA TCA CT) and MAR-251R (5' CAA AGA TGT CCT TGG GTG TG), designed based on conserved regions of the amino acid sequence of the putative transposase gene of the Mariner element [74]. The PCR products were cloned using pGEMT kit (Promega, Madison, WI, USA) according to manufacturer's recommendations. The recombinant plasmids were submitted to nucleotide sequencing using a sequencer model 3500 Genetic Analyzer (Applied Biosystems, Foster City, CA, USA).

The DNA sequences obtained were used as an initial query to searches against a database of repetitive DNA elements (Repbase database) (http://www.girinst.org/ repbase/), which contains repetitive DNA sequences of various eukaryotic species [75]. Additionally, the obtained sequences were analyzed against the nucleotide collection of The National Center for Biotechnology (NCBI) (http:// www.ncbi.nlm.nih.gov) using the Blast search tool. Family consensus sequences were constructed whenever possible. The analysis of DNA sequences were performed with web site LIRMM (Laboratoire Le d'Informatique, Robotique et de Microélectronique of Montpellier), available online at http://www.phylogeny.fr/ [68,76,77]. The multiple alignments were performed using MUSCLE, while the alignment curation used Gblocks program to eliminate poorly aligned positions and divergent regions. Phylogenetic trees were built with neighbor joining (NJ) and confirmed as consistent with trees built by PhyML [78]. The Mariner protein coding sequences were searched against the Pfam database (http://pfam.sanger.ac.uk). For comparison, was also prepared a phylogenetics analysis using the Maximum Likehood, measuring the consistency by bootstrap using the program MEGA5 - Molecular Evolutionary Genetics Analysis (http://www.megasoftware.net/).

\section{Chromosome preparation and Fluorescence in situ hybridization (FISH)}

Meiotic chromosomes for FISH were obtained from testes of Coprophanaeus cyanescens, C. ensifer and Diabroctis mimas according to the classic technique of squashing of testicular follicles using a drop of $45 \%$ acetic acid, and then dipped in liquid nitrogen to remove the coverslip.

The PCR products containing a pool of Mariner sequences, and the $C_{0} t-1$ DNA fraction were labeled with biotin-11-dATP by nick translation using the Bionick Labeling System kit (Invitrogen, San Diego, CA, USA). The FISH protocol followed the adaptations described by Cabral-de-Mello et al. [79]. The probes were labeled with biotin-14-dATP and detected by avidin-FITC (fluorescein isothiocynate) conjugated (Sigma-Aldrich, St. Louis, MO, USA). The chromosomes were counterstained with 4,6- 
diamidino-2-phenylindole (DAPI) and the slides mounted with Vectashield (Vector, Burlingame, CA, USA). The images were captured using Olympus DP71 digital camera coupled to a BX61 Olympus microscope and DP Control program, and processed through Corel Photo-Paint 12 and Adobe Photoshop CS2.

\section{Additional files}

Additional file 1: Figure S1. Cross-species hybridization of $C_{0} t-1$ DNA fraction in metaphases I of Coprophanaeus species. Probe of $C$. ensifer hybridized in C. cyanescens (a) and probe of Coprophanaeus cyanescens hybridized in C. ensifer (b). Bar $=5 \mu \mathrm{m}$.

Additional file 2: Dataset S1. Sequence alignment of related Mariner families of diverse organisms retrieved from public databases and sequences obtained in the present work. The abbreviations correspond to the species names and IDs, as shown in the caption of Figure 3. Dashes represent indels.

Additional file 3: Figure S2. Alignment guide tree of Mariner families based on Maximum Likehood. The taxa are the same as described in Figure 3 . The bootstrap support values are indicated on the nodes. The scale bar indicates the genetic distance.

Additional file 4: Dataset S2. Alignments pairwise similarity matrix of related Mariner families. The abbreviations correspond to the species names and IDs, as shown in Figure 3.

\section{Competing interests}

The authors declare that they have no competing interests.

\section{Authors' contributions}

SGO carried out the cytogenetic and molecular analysis and draft the manuscript DCC helped in obtained cytogenetic data. RCM, CM and DCC conceived of the work, and participated in its design, drafted and revised the manuscript. All authors analyzed all results and read and approved the final manuscript.

\section{Acknowledgements}

The authors are grateful to CMQ Costa and FAB Silva for the taxonomic identification of the species studied. The study was supported by the Coordenadoria de Aperfeiçoamento de Pessoal de Nível Superior (CAPES), the Conselho Nacional de Desenvolvimento Científico e Tecnológico (CNPq), the Fundação de Amparo a Pesquisa do Estado de São Paulo (FAPESP, grants 2008/02830-2, 2009/04351-7, 2013/04533-3), and the Fundação de Amparo a Ciência e Tecnologia do Estado de Pernambuco (FACEPE).

\section{Author details}

${ }^{1}$ Morphology Department, Biosciences Institute, UNESP - São Paulo State University, Botucatu, SP 18618-970, Brazil. ${ }^{2}$ Biology Department, Biosciences Institute, UNESP - São Paulo State University, Rio Claro, SP 13506-900, Brazil. ${ }^{3}$ Department of Biology, Biological Sciences Institute, UPE - Pernambuco State University, Recife, PE 50100-130, Brazil.

Received: 6 September 2013 Accepted: 25 October 2013 Published: 29 November 2013

\section{References}

1. Dimitri P, Junakovic N: Revising the selfish DNA hypothesis: new evidence on accumulation of transposable elements in heterochromatin. Trends Genet 1999, 4:123-124.

2. Torti C, Gomulski LM, Moralli D, Raimondi E, Robertson HM, Capy P, Gasperi $G$, Malacrida AR: Evolution of different subfamilies of mariner elements within the medfly genome inferred from abundance and chromosomal distribution. Chromosoma 2000, 108:523-532.

3. Pikaard C, Pontes O: Heterochromatin: condense or excise. Nature Cell Biol 2007, 9:19-20

4. Montiel EE, Cabrero J, Camacho JPM, López-León MD: Gypsy, RTE and Mariner transposable elements populate Eyprepocnemis plorans genome. Genetica 2012, 140:365-374.
5. Kazazian HH Jr: Mobile elements: drivers of genome evolution. Science 2004, 303:1626-1632.

6. Feschotte C: Transposable elements and the evolution of regulatory networks. Nature Rev Genet 2008, 9:397-405.

7. Pritham EJ: Transposable elements and factors and their success in eukaryotes. J Hered 2009, 100:648-655.

8. Walisko $O$, Jursch T, Izavák Z, Ivics Z: Transposon-host cell interactions in the regulation of Sleeping Beauty transposition. In Transposons and the Dynamic Genome. Edited by Lankenau D-H, Volff J-N. Berling Heidelberg: Springer-Verlag; 2009:109-132.

9. Robertson HM: The TC1-mariner superfamily of transposons in animals. J Insect Physiol 1995, 41:99-105.

10. Wicker T, Sabot F, Hua-Van A, Bennetzen JL, Capy P, Chalhoub B, Flavell A, Leroy P, Morgante M, Panaud O, Paux E, SanMiguel P, Schulman AH: A unified classification system for eukaryotic transposable elements. Nat Rev Genet 2007, 8:973-982.

11. Lampe DJ, Churchill MEA, Robertson HM: A purified mariner transposase is sufficient to mediate transposition in vitro. EMBO J 1996, 15:5470-5479.

12. Plasterk RH: The Tc1/mariner transposon family. Curr Top Microbiol Immunol 1996, 204:125-143.

13. Hartl DL, Lozovskaya ER, Nurminsky DI, Lohe AR: What restricts the activity of mariner -like transposable elements? Trends Genet 1997, 13:197-201.

14. Robertson HM: The mariner transposable element is widespread in insects. Nature 1993, 362:241-245.

15. Miskey C, Izsvák Z, Kawakami K, Ivics Z: DNA transposons in vertebrate functional genomics. Cell Mol Life Sci 2005, 62:629-641.

16. Feschotte C, Pritham EJ: DNA Transposons and the evolution of eukaryotic genomes. Annu Rev Genet 2007, 41:331-368.

17. Robertson HM, Lampe DJ: Recent horizontal transfer of a mariner transposable element among and between Diptera and Neuroptera. Mol Biol Evol 1995, 12:850-862.

18. Ray DA, Feschotte C, Pagan HJT, Smith JD, Pritham EJ, Arensburger P, Atkinson PW, Craig NL: Multiple waves of recent DNA transposon activity in the bat, Myotis lucifugus. Genome Res 2008, 18:717-728.

19. Oliveira SG, Bao W, Martins C, Jurka J: Horizontal transfers of Mariner transposons between mammals and insects. Mobile DNA 2012, 3:14.

20. Sormacheva I, Smyshlyaev G, Mayorov V, Blinov A, Novikov A, Novikovaz O: Vertical evolution and horizontal transfer of CR1 non-LTR Retrotransposons and Tc1/mariner DNA transposons in Lepidoptera species. Mol Biol Evol 2012, 29:3685-3702.

21. Lim JK, Simmons MJ: Gross chromosome rearrangements mediated by transposable elements in Drosophila melanogaster. Bioessays 1994, 16:269-275.

22. Gray YHM: It takes two transposons to tango - transposable-elementmediated chromosomal rearrangements. Trends Genet 2000, 16:461-468.

23. Lonnig W-E, Saedler H: Chromosome rearrangements and transposable elements. Annu Rev Genet 2002, 2002(36):389-410.

24. Oliveira SG, Moura RC, Martins C: B chromosome in the beetle Coprophanaeus cyanescens (Scarabaeidae): emphasis in the organization of repetitive DNA sequences. BMC Genet 2012, 13:96.

25. Bione E, Camparoto ML, Simões ZLP: A study of the constitutive heterochromatin and nucleolus organizer regions of Isocopris inhiata and Diabroctis mimas (Coleoptera: Scarabaeidae, Scarabaeinae) using C-banding, $\mathrm{AgNO}_{3}$ staining and FISH techniques. Gen Mol Biol 2005, 28:111-116.

26. Louzada JN: Scarabaeinae (Coleoptera: Scara- baeidae) detritívoros em ecossistemas tropi- cais: biodiversidade e serviços ambientais. In Biodiversidade do Solo em Ecossistemas Brasileiros. Edited by Moreira FMS, Siqueira JO, Brussaard L L. Lavras: UFLA; 2008:309-332.

27. Oliveira SG, Moura RC, Silva AEB, Souza MJ: Cytogenetic analysis of two Coprophanaeus species (Scarabaeidae) revealing wide constitutive heterochromatin variability and the largest number of $45 \mathrm{~S}$ rDNA sites among Coleoptera. Micron 2010, 41:960-965.

28. Schweizer D, Loidl J: A model for heterochromatin dispersion and the evolution of C- bands patterns. Chromosome Today 1987, 9:61-74.

29. Sumner AT: Chromosomes: organization and function. Blackwell Science Ltd: North Berwich; 2003.

30. Loreto V, Stadtler E, Melo NF, Souza MJ: A comparative cytogenetic analysis between the grasshopper species Chromacris nuptialis and C. speciosa (Romaleidae): constitutive heterochromatin variability and rDNA sites. Genetica 2005, 125:2530-260.

31. Cabral-de-Mello DC, Moura RC, Martins C: Chromosomal mapping of repetitive DNAs in the beetle Dichotomius geminatus provides the first 
evidence for an association of $5 \mathrm{~S}$ rRNA and histone $\mathrm{H} 3$ genes in insects, and repetitive DNA similarity between the $\mathrm{B}$ chromosome and $\mathrm{A}$ complement. Heredity 2010, 104:393-400.

32. Juan C, Petitpierre E: C-banding and DNA content in seven species of Tenebrionidae (Coleoptera). Genome 1989, 32:834-839.

33. Rozek M, Lachwska D, Petitpierre E, Holecová M: C-bands on chromosomes of 32 beetles species (Coleoptera: Elateridae, Cantharidae, Oedemeridae, Cerambycidae, Chrysomelidae and Curculionidae). Hereditas 2004, 140:201-211.

34. Angus RB, Wilson CJ, Mann DJ: A chromosomal analysis of 15 species of Gymnopleurini, Scarabaeini and Coprini (Coleoptera: Scarabaeidae). Tijdsch voor Entomol 2007, 150:201-211.

35. Cabral-de-Mello DC, Moura RC, Melo AS, Martins C: Evolutionary dynamics of heterochromatin in the genome of Dichotomius beetles based on chromosomal analysis. Genetica 2011, 139:315-325.

36. Charlesworth B, Snlegowskl P, Stephan W: The evolution dynamics of repetitive DNA in eukaryotes. Nature 1994, 371:215-220.

37. Li YC, Kord AB, Fahima T, Berles A, Nero E: Microsatellites: genomic distribution, putative functions and mutation mechanisms: a review. Mol Ecol 2002, 11:2453-2465.

38. Shapiro JA, Sternberg R: Why repetitive DNA is essential to genome function. Biol Rev 2005, 80:1-24.

39. Kaminker JS, Bergman CM, Kronmiller B, Carlson J, Svirskas R, Patel S, Frise E, Wheeler DA, Lewis SE, Rubin GM, Ashburner M, Celniker SE: The transposable elements of the Drosophila melanogaster euchromatin: a genomics perspective. Genome Biol 2002, 3:1-20.

40. Delaurière L, Chénais B, Hardivillier Y, Gauvry L, Casse N: Mariner transposons as genetic tools in vertebrate cells. Genetica 2009, 137:9-17.

41. Cabral-de-Mello DC, Oliveira SG, Ramos IC, Moura RC: Karyotype differentiation patterns in species of the subfamily Scarabaeinae (Scarabaeidae, Coleoptera). Micron 2008, 39:1243-1250.

42. Deininger PL, Moran JV, Batzer MA, Kazazian HH Jr: Mobile elements and mammalian genome evolution. Curr Opin Genet Dev 2003, 13:651-658.

43. Chen JM, Stenson PD, Cooper DN, Ferec C: A systematic analysis of LINE-1 endonuclease-dependent retrotranspositional events causing human genetic disease. Hum Genet 2005, 117:411-427.

44. Slotkin RK, Martienssen R: Transposable elements and the epigenetic regulation of the genome. Nat Rev Genet 2007, 8:272-285.

45. Dimitri $P$, Arcà $B$, Berghella $L$, Mei E: High genetic instability of heterochromatin after tranposition of the LINE-like I factor in Drosophila melanogaster. Proc Natl Acad Sci USA 1997, 94:8052-8057.

46. von Stenberg RM, Novick GE, Gao G-P, Herrera RJ: Genome canalization: the coevolution of transposable and interspersed repetitive elements with single copy DNA. Genetica 1992, 86:106-137.

47. McDonald JF: Evolution and consequences of transposable elements. Curr Opin Genet Develop 1993, 3:855-864.

48. Dawe RK: RNA interference, transposons, and the centromere. Plant Cell 2003, 15:297-301.

49. Wong LH, Choo KH: Evolutionary dynamics of transposable elements at the centromere. Trends Genet 2004, 20:611-616.

50. Steinemann M, Steinemann S: Degenerating Y chromosome of Drosophila miranda: a trap for retrotransposons. Proc Natl Acad Sci USA 1992, 89:7591-7595.

51. Mandrioli M: Identification and chromosomal localization of Mariner-like elements in the cabbage moth Mamestra brassicae (Lepidoptera). Chromosome Res 2003, 11:319-322.

52. Bachtrog D: Sex chromosome evolution: Molecular aspects of $Y$ chromosome degeneration in Drosophila. Genome Res 2005, 15:1393-1401.

53. Charlesworth D, Charlesworth B, Marais G: Steps in the evolution of heteromorphic sex chromosomes. Heredity 2005, 95:118-128.

54. Langley CH, Montgomery E, Hudson R, Kaplan N, Charlesworth B: On the role of unequal exchange in the containment of transposable element copy number. Genet Res 1988, 52:223-235.

55. Junakovic N, Terrinoni A, Di Franco C, Vieira C, Loevenbruck C: Accumulation of transposable elements in the heterochromatin and on the $\mathrm{Y}$ chromosome of Drosophila simulans and Drosophila melanogaster. J Mol Evol 1998, 46:661-668.

56. Gomulski LM, Brogna S, Babaratsas A, Zacharopoulou A, Savakis C, Bourtzis $\mathrm{K}$ : Molecular basis of the size polymorphism of the first intron of the Adh-1 gene of the Mediterranean fruit fly, Ceratitis capitata. J Mol Evol 2004, 58:732-742.
57. Capy P, Vitalis R, Langin T, Higuet D, Bazin C: Relationships between transposable elements based upon the integrase-transposase domains: is there a common ancestor? J Mol Evol 1996, 42:359-368.

58. Plasterk RHA, Izsvák Z, Ivics Z: Resident aliens: the Tc1/mariner superfamily of transposable elements. Trend Genet 1999, 15:326-332.

59. Robertson HM, Lampe DJ: Distribution of transposable elements in arthropods. Annu Rev Entomol 1995, 40:333-357.

60. Robertson HM, MacLeod EG: Five major subfamilies of Mariner transposable elements in insects, including the Mediterranean fruit fly, and related arthropods. Insect Mol Biol 1993, 2:125-139.

61. Robertson HM, Zumpano KL: Molecular evolution of an ancient mariner transposon, Hsmar1, in the human genome. Gene 1997, 205:203-217.

62. Kordis D, Gubensek F: Unusual horizontal transfer of a long interspersed nuclear element between distant vertebrate classes. Proc Natl Acad Sci USA 1998, 95:10704-10709.

63. Piskurek O, Okada N: Poxviruses as possible vectors for horizontal transfer of retroposons from reptiles to mammals. Proc Natl Acad Sci USA 2007, 104:12046-12051.

64. Gilbert C, Pace JK, Feschotte C: Horizontal SPINning of transposons. Commun Integr Biol 2009, 2:1-3.

65. Schaack S, Gilbert C, Feschotte C: Promiscuous DNA: horizontal transfer of transposable elements and why it matters for eukaryotic evolution. Trends Ecol Evol 2010, 25:537-546.

66. Pace JK II, Gilbert C, Clark MS, Feschotte C: Repeated horizontal transfer of a DNA transposon in mammals and other tetrapods. Proc Natl Acad Sci USA 2008, 105:17023-17028.

67. Böhne A, Brunet F, Galiana-Arnoux D, Schultheis C, Volff J-N: Transposable elements as drivers of genomic and biological diversity in vertebrates. Chromosome Res 2008, 16:203-215.

68. Dereeper A, Guignon V, Blanc G, Audic S, Buffet S, Chevenet F, Dufayard JF, Guindon S, Lefort V, Lescot M, Claverie JM, Gascuel O: Phylogeny.fr: robust phylogenetic analysis for the non-specialist. Nucleic Acids Res 2008, 36:465-469.

69. Kidwell M: Lateral transfer in natural populations of eukaryotes. Annu Rev Genet 1993, 27:235-256.

70. Silva JC, Kidwell MG: Horizontal transfer and selection in the evolution of P elements. Mol Biol Evol 2000, 17:1542-1557.

71. Sambrook J, Russel DW: Molecular Cloning. A Laboratory Manual. 3rd edition. Cold Spring Harbor: Cold Spring Harbor Laboratory Press; 2001.

72. Zwick MS, Hanson RE, McKnight TD, Nurul-Islam-Faridi M, Stelly DM: A rapid procedure for the isolation of $C_{0} t-1$ DNA from plants. Genome 1997, 40:138-142.

73. Ferreira IA, Martins C: Physical chromosome mapping of repetitive DNA sequences in Nile tilapia Oreochromis niloticus: evidences for a differential distribution of repetitive elements in the sex chromosomes. Micron 2008, 39:411-418

74. Lampe DJ, Witherspoon DJ, Soto-Adames FN, Robertson HM: Recent horizontal transfer of mellifera subfamily Mariner transposons into insect lineages representing four different orders shows that selection acts only during horizontal transfer. Mol Biol Evol 2003, 20:554-562.

75. Jurka J, Kapitonov W, Pavlicek A, Klonowski P, Kohany O, Walichiewicz J: Repbase Update, a database of eukaryotic repetitive elements. Cytogenet Genome Res 2005, 110:462-467.

76. Guindon S, Gascuel O: A simple, fast, and accurate algorithm to estimate large phylogenies by maximum likelihood. Syst Biol 2003, 52:696-704.

77. Chevenet F, Brun C, Banuls AL, Jacq B, Christen R: TreeDyn: towards dynamic graphics and annotations for analyses of trees. BMC Bioinformatics 2006, 7:439.

78. Guindon S, Dufayard JF, Lefort V, Anisimova M, Hordijk W, Gascuel O: New algorithms and methods to estimate maximum-likehood phylogenies: assessing the performance of PhyML 3.0. Syst Biol 2010, 59:307-321.

79. Cabral-de-Mello DC, Moura RC, Carvalho R, Souza MJ: Cytogenetic analysis of two related Deltochilum (Coleoptera, Scarabaeidae) species: diploid number reduction, extensive heterochromatin addition and differentiation. Micron 2010, 41:112-117.

doi:10.1186/1755-8166-6-54

Cite this article as: Oliveira et al.: Chromosomal organization and evolutionary history of Mariner transposable elements in Scarabaeinae coleopterans. Molecular Cytogenetics 2013 6:54. 